\title{
Markers and models to personalize cancer therapy
}

\section{$\underline{\text { Sidransky D. }}$}

Johns Hopkins University, Baltimore, USA

Background: Clonal genetic alterations including gene mutations, epigenetic alterations, or mitochondrial changes are a hallmark of human cancer. These genetic alterations can be detected in clinical samples using DNA amplification techniques and used to estimate tumor burden and for the early detection of cancer. Moreover, these alterations can be tested by molecular techniques in clinical trials materials to provide better estimates of prognosis and prediction of therapy. Individualized xenograft models can also help integrate such markers and help define new combinations of novel and standard drugs. These models can also be used as avatars to test standard and experimental drugs before using these treatments on the actual patients in the clinic. Biomarkers of sensitivity and resistance will emerge from these models and will help further guide personalized therapy with more success and less toxicity in larger populations. 